\title{
Performance Analysis of Remote Sensing Application using Area Wise Prediction
}

\section{K. VIJAYALAKSHMI and V. VIJAY KUMAR*}

SNIST, Yamnampet, Ghatkesar, Hyderabad-501301, India.

\section{Abstract}

Remote sensors from the Satellite or Aircrafts are generated by huge volume of data which can utilize for impending signification if collected data aggregated effectively incorporates by insight information. Data is collection from simple to hybrid devices, which are continuously working for technology around us and communicate with each other. These devices are transferring huge amounts of real time data daily. The transaction added to the synchronized inaccessible sensing data that is retrieving the useful information in the proficient way of classification in the direction of the severe computational challenges, analyze, the assortment, and accumulate, where gathered data is inaccessible. The real time sensing devices will continuously export data. In this work, we will implement the big data analytics on remote sensing datasets. We utilized BEST software for header analysis of the datasets and retrieving the full resolution image from the dataset. Then retrieved image is divided into smaller blocks for applying statistical. By applying certain rules and conditions in the form of algorithm, determine the land and sea blocks of image dataset. Our end results are proficiently analyzing real-time remote sensing utilizing the land beacon structure. Finally, a comprehensive investigation of the remotely intelligence earth beacon massive information for earth and ocean space are available by utilizing- Hadoop.

\section{Introduction}

The expression "remote sensing satellites" is also called as the "aerial photography" regard as the depiction. The main research focused on the evolution of remote sensing satellites in four decades i.e., 1960-2010. Detecting the portraits of the huge plane from expanded partitions utilized airborne picture making and they utilized to grab the pictures. ${ }^{1}$ Remote sensing systems came into the photography and they have been fetched in satellites for depiction

CONTACT V. Vijay Kumar $\lesssim$ vldms@yahoo.com Sreenidhi Institute of Science and Technology, Yamnampet, Ghatkesar, Hyderabad, Telangana, India.

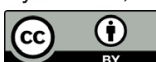

(C) 2018 The Author(s). Published by Oriental Scientific Publishing Company

This is an $\partial$ Open Access article licensed under a Creative Commons license: Attribution 4.0 International (CC-BY).

Doi: http://dx.doi.org/10.13005/ojcst12.01.05 
obtaining. To compare with planes, satellites cover ${ }^{2}$ more surface regions and there are widely utilized.

Remotely detected symbolism from satellites ${ }^{3}$ - investigate and upgraded with PCs - made it conceivable to recognize and screen these progressions. Only some groups are exceptional with the term remote sensing.

Remote satellites continuously obtaining high resolution pictures of the earth's surface on the base stations. Data will take care of base stations in running several partitions stepwise. These partitions process the data. After gathering data, it is preprocessing so as to eliminate the noisy elements in the data to obtain high throughput. The data transformed into pictures for the purpose of being comprehensible formats and these images can be analyzed. By utilizing parameters by pertaining the threshold provisions to the depictions will partition the picture into the number of blocks which can be analyzed easily processing in making decision and promoting. Then we analyzed the predicted regions from the land surface.

\section{Related Work}

RS (Remote sensors) is utilized for ground observatory and streaming the continuous data and creates an extent of data. Many researchers implemented diverse areas of applications in satellite remote sensing data, for instance, the gradient based edge detection, ${ }^{4}$ transformation, identification and so on. The continuous real time streaming data ${ }^{5}$ with high speed or huge extent of data available in the form of big data which lead to a many challenges? In this regard, transformation of the sensor data from remote places are difficult to scientific understanding is challenging task. Satellite remote sensing is utilizing of various technologies to construct scrutiny and dimensions of destination that is usually recognizable to the stripped eye. The technologies in remote sensing are classified as, radar, thermal, seismic, sonar, LiDAR, electric field sensing, GPS and infrared radiation. The remote sensing satellites will export the data in the form of offline and online real time data

Many researchers developed models based on satellite data for coastline analysis and erosion.
For example, shoreline changing rates, erosion of coastal and accusation in coastal of Tamil Nadu 6 utilized satellite data. GIS tools ${ }^{7}$ are used to analyze Multitemporal remote sensing data from 1976 to 2000 and twenty scenes are taken for changing pattern of accretion and erosion of sub aerial delta of Yellow River. Black and white aerial photographs ${ }^{8}$ are utilized to estimating the shoreline change rate in Neuse River Estuary, USA. Space simulation ${ }^{9}$ is introducing a new way of understanding the geographical space which includes the dynamic processes in the space analysis.

\section{Framework \\ Evaluation Methods \\ Dataset}

Dataset is utilized in this work from "European space agency" which contains the number of tasks that are executed in the earlier period. And also took the necessary data for implementation and to examine the complex parameters for evaluation. After preprocessing, datasets will be added to description. Then this data set is classified into three main fields. The entire products are classified beneath of Advanced Synthetic Aperture Radar (ASAR).

\section{Category 1: Agency of European}

ASAR product optional domain is APM (Alternating Polarization Mode) and it includes both Coregistered pictures belongs to one of the three divergence amalgamation secondary modes (VV and $\mathrm{VH}, \mathrm{HH}$ and $\mathrm{VV}, \mathrm{HH}$ and $\mathrm{HV}$ ). In addition, the result utilizes the Range Doppler (RD) algorithm and preprocessing data parameters accessible. It can be activated to infer added animated items for SAR account superior appraisal, alignment, and interferon metric applications, if accustomed of the device attainment. The entire products will be in the structure of (.N1) extension categorizer design.

Preprocessing data: In this phase, cleaning the data for removing the unwanted elements. The authentic preprocessing techniques consist of header analysis, missing values and noise elements. These datasets having will .HAN extension files. Given dataset is inputted to header analysis and it converting the dataset into picture format. For every time, it will carry on producing the set of documents that sustain the data retrieval. 
HAN documentation contains investigation and restrictions of the datasets. This documentation contains size, largeness and magnitude in pixels, dimensions and constraints like SPH indicators, etc. After header observation completed the properties of the datasets are able to understand and the dimensions of the datasets will be utilized for partitioning the picture into an amount of chunks.
Full resolution pictures of the dataset are.N1 form in .XT documents. The information is set up to be export in the form of understandable for humans, which looks like GeoTIFF, TIFF picture formats. Preferring any of the result's format (for our experiment results we utilized TIFF) and sending the picture in needed directory file. A fundamental fault occurs if the time concerns and continuous without coordinating

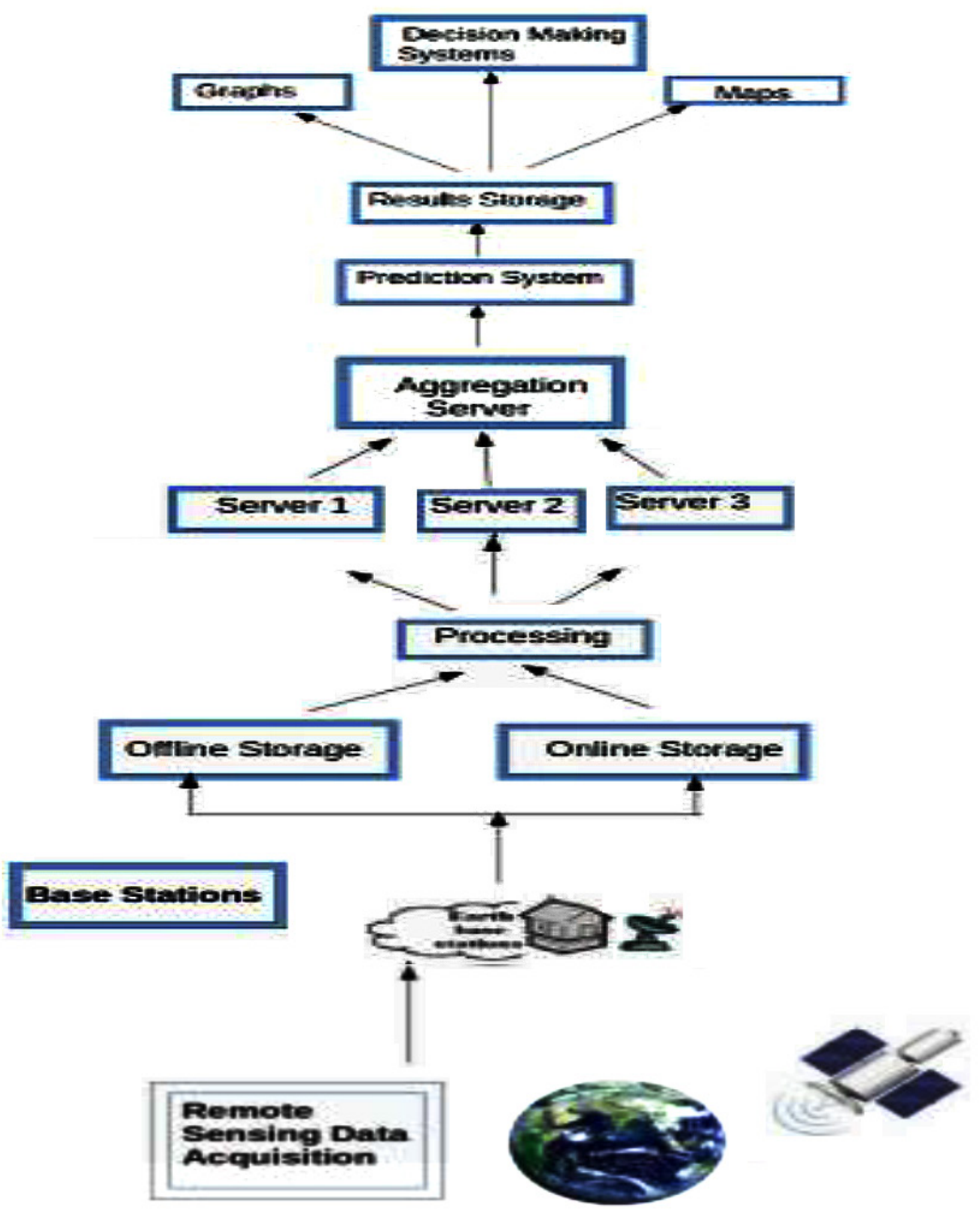

3.1. Framework of remote sensing application 
dataset. Beside the picture, it furthermore generates the accurate restrictions of the datasets.

\section{Domain 2: Resolution Image Mode Medium}

When the device was in picture mode the data accumulated at Level zero ASAR-IMM created. By comparing with ASA_IMP, this result has lesser resolution but elevated radiometric resolution which is adequate for ice applications which apply to a continuous area besides the imaging swath and features a radiometric resolution (ENL). Preprocessing is same as the domain 1.

\section{Domain 3: Image in Global Monitoring Mode}

When the device was in global monitoring mode the data accumulated at Level zero ASAR-GM1 created. Each item covers an entire trajectory. The research work includes slant range to land modifications. Standard for ASAR Global Monitoring Mode is the strip-line product. By utilizing the SPECAN algorithm it processed to one $\mathrm{km}$ resolution approximately.

\section{Methodology}

In our proposed method, the image which is retrieved from the dataset is partitioning into a small blocks for analyzing the geometric parameters. The partition is done due to come across the precise information. For every chunk, identifying the statistical parameters and assessment analysis is prepared from blocks. In this work, every image is partitioned into 20 blocks; these blocks are utilized for accurate analysis.

The bunch of chunks derived from the picture will be using as input for the proposed algorithm. The algorithm acquires every chunk of the picture and updates the image list. This list is utilized diverse methods and functions for the purpose of analyze the statistical parameters of every chunk. For every picture chunk, the algorithm determines absolute difference, mean, standard deviation, etc.

For estimating decision analysis process, the major parameters are:

Table 3.1. Data set of three major domains

\begin{tabular}{|c|c|c|c|}
\hline Domian & /Dataset Details & I/P Dataset Details & Final Dataset Output \\
\hline \multirow[t]{2}{*}{ ASA_APM } & Alternating & APM uses Range Doppler & ASA_APM. XT \\
\hline & Polarization Mode & $\begin{array}{l}\text { Algorithm used to drive higher } \\
\text { level products for SAR image } \\
\text { quality assessment, calibration, } \\
\text { and interferometric applications, }\end{array}$ & converted to image \\
\hline \multirow[t]{2}{*}{ ASA_IMM } & Image Mode & IMM uses Covers a continuous & ASA_IMM. XT \\
\hline & $\begin{array}{l}\text { Medium Resolution } \\
\text { Image }\end{array}$ & $\begin{array}{l}\text { area along the imaging swath and } \\
\text { features an ENL (radiometric } \\
\text { resolution good enough for ice } \\
\text { applications. }\end{array}$ & converted to image \\
\hline ASA_GM1 & Global Monitoring & GM1 is processed to approximately $1 \mathrm{Km}$ & ASA_GM1.XT \\
\hline
\end{tabular}

Table 4.1. Maximum and minimum of parameters

\begin{tabular}{lccccccc}
\hline $\begin{array}{l}\text { Product/ } \\
\text { Dataset }\end{array}$ & Max_mean & Min_mean & Max_std & Min_std & Max_abs diff & min_abs diff \\
\hline ASA_APM & 43.3173687776 & 1.86531039356 & 10.4488577999 & 0.391309719305 & 0.391309719305 & 1.40931380522 \\
ASA_IMM & 126.605075138 & 0.616355714003 & 40.7494092775 & 0.0604999073497 & 85.5566586 & 0.130082799048 \\
ASA_GM1 & 76.2293183657 & 2.6266050089 & 33.1323265705 & 0.637487603441 & 48.5039592165 & 1.98911740546 \\
\hline
\end{tabular}


1. Xi (Mean) 2. SD (Standard Deviation) 3. AD (Absolute difference)

The variables and parameters utilized in our proposed algorithms.

Fixed size chunks of Picture are B1, B2, B3, B4, B5 ... BN $\backslash$.

Number of lines in the Image $=$ NR.

Number of pixels in every line $=$ NSR

Block of image size $=$ Total number of chunks in the picture $=\mathrm{N}(\mathrm{N}=\mathrm{NR} \times \mathrm{NSR})$

BS

Mean of model values of chunk $\mathrm{BI}=\mathrm{X}$ Bi where $\mathrm{i}=$ $\{1,2,3,4 \ldots N\}$.

Summation of all values of the chunk $B$ i /the size of chunk $=\mathrm{X} \mathrm{Bi}$

$\mathrm{SD}$ of sample values of chunk $\mathrm{Bi} .=\mathrm{SD} B \mathrm{Bi}$

$A B$ between $X \mathrm{Bi}$ and $\mathrm{SD} B \mathrm{Bi}=$ Abs_Diff

| X Bi - SD Bi I = Abs Diff

Total chunks mean $\mathrm{Bi} /$ the total number of chunks = Centric mean
By means of the above mention limitations, the subsequent will help in constructing the provisions and decision making.

The upper limit mean from mean of chunks is known as Maximum mean

The maximum SD from SD of chunks is known as Max SD.

Maximum $A D$ from $A D$ of chunks is known as Max AD

Minimum AD from AD of chunks is known as Min AD. Mean of all the chunks /Number of chunks = Centric mean

SD of all the chunks = Centric SD Number of chunks For simplifying the restrictions, the in detail conditions are identified. Those:

Mean less than Centric mean = MLCM

Mean greater than centric mean $=$ MGCM

SD less than centric SD = SLCS

SD greater than centric SD = SGCS

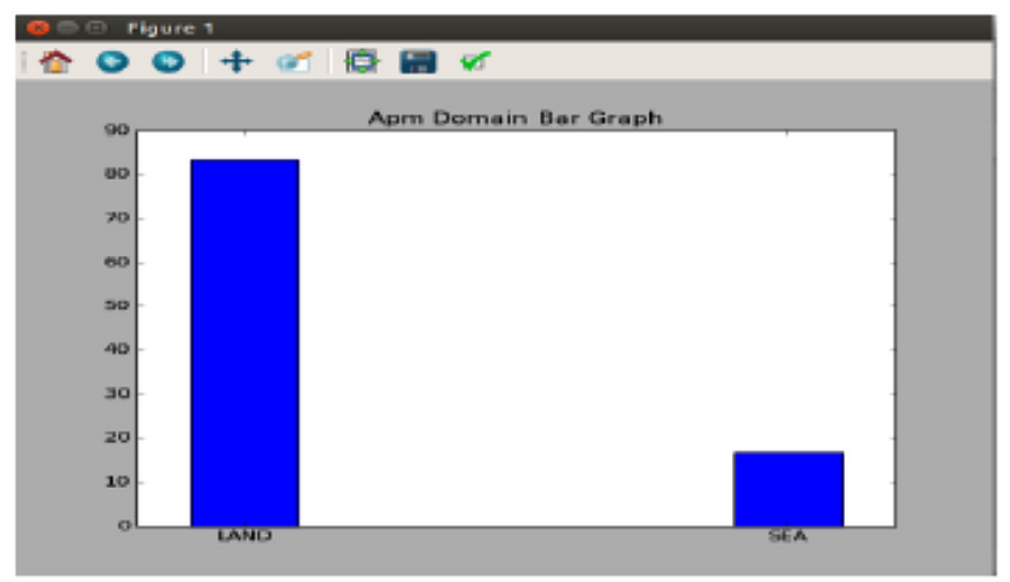

Fig. The output of land and sea percentages in the bar graph representation

\section{S. No. Product Domain Product/ Dataset Number No. of blocks}

\begin{tabular}{cccc}
\hline 1 & ASA_AMP & 10 & 10 20=200 \\
2 & ASA_Imm & 10 & 200 \\
3 & ASA_GM1 & 10 & 200 \\
\hline
\end{tabular}


$A D$ less than centric $A D=A L C A$

$A D$ greater than centric $A D=A G C A$

In our approach, we build the following laws for detection of the region

1. Centric mean $>=$ Mean of $\mathrm{Bi}$

2. Centric $\mathrm{SD}<=\mathrm{SD}$ of $\mathrm{Bi}$

3. $\mathrm{AD}$ of $\mathrm{Bi}<=$ Centric $\mathrm{AD}$

For every $(\mathrm{Bi})\{$

If (law1 == true and law2 == true)

Condition_Bi $=$ earth

Else if (law1 $==$ false andlaw2 $==$ false)

Condition_Bi $=$ ocean

Else \{

If (law3 == false and law1 == false)

Condition_Bi $=$ ocean

Else

Condition_Bi = earth\}

For identifying the earth chunks

1. Centric mean $>=$ Mean of $\mathrm{Bi}$

2. SD of $\mathrm{Bi}>=$ Centric SD

Or

1. Centric mean $>=$ Mean of $\mathrm{Bi}$

2. $A D$ of $B i>$ Centric $A D$

Or

1. Centric mean $<$ Mean of $B i$

2. $A D$ of $B i>$ Centric $A D$

For identifying the ocean chunks

1. Centric mean $<$ Mean of $\mathrm{Bi}$

2. $\mathrm{SD}$ of $\mathrm{Bi}<$ Centric $\mathrm{SD}$

3. $A D$ of $B i>$ Centric $A D$

\section{Visualization of Data}

The blocks are able to understand that whether they belong to land or sea regions after applying above rules for blocks of image. In our implementation, we take .csv file belongs to the land blocks and sea blocks for the better visualization of data. For this we utilized the python programming and done visualization for the obtained blocks based on the mean of the blocks. The packages in python programming are pandas, Numpy, matplotlib and seaborne which are utilizing for statistical analysis and understandable visualization.
For example, the land and sea percentages for domain Alternating Polarization Medium is Percentage of Land is 83.3710954415

Percentage of Sea is 16.6289045585

\section{Analysis of Performance}

We estimate the performance of proposed method by increasing the number of chunks size and predicting the surface area region of land area or sea area.

In our performance analysis testing, IMM products has $86 \%$ land region than the other products which proves that highest land region has less sea region. With this we conclude that IMM has $13.9 \%$ sea region where the other products APM and GM1 has land ranging from $78-83 \%$ and sea $16-22$ $\%$. The table takes the 25 images as input and each produces 20 smaller divided partitions. Each partition will give $20^{\star} 25=500$ chunks which will be used for predicting the surface region.

By calculating performance analysis, IMM gives more land region when compared with other products/ IMM has $84 \%$ land region and $15.4 \%$ sea region whereas the APM and GM1 has land ranging from $77-83 \%$ and sea $16-23 \%$.

The table indicates the performance testing three that the GM1 has $85 \%$ land region (than IMM product and $14.9 \%$ of sea region whereas the APM and IMM products has land ranging from $77-84 \%$ and sea $16-23 \%$.

Global Monitoring Mode (GM1) is increasing the number of partitioning chunks which gives more land region and less sea region. Whereas APM (Alternating Polarization Mode) product maintains the constant percentage of land region as well as the sea region and it producing large amount of variation between percentages of regions is not seen in three performance testing made.

In case of Image Mode Medium Resolution Image (IMM) the land percentages are decreasing as increasing the number of chunks. As the number of images in the domain of IMM is used for performance testing gives the gradual decrease in land and sea regions. 


\section{Conclusions}

In this work, we analyze the dissimilar domains in the remote sensing satellites which are uninterruptedly releasing the data. With the different types of products are increasing we observed and analyzed the data behave with regard to land and sea surface regions. The data from satellite emitting continuously, surface area analysis will be less in time when different areas with diverse products are in use and analyze the surface regions. For analysis of large number of surface regions required high processing computational devices for ex, if we take 10 surface regions will require more than 500 products for each domain.

\section{Future Work}

In further, we will extend our work not only for predicting and identify the land and sea regions but also include diverse areas like forest areas, sand areas etc. we extending this work for predicting the earthquakes, tsunamis and natural disasters that can be recognized with analysis of proper recommendation to avoid the natural disasters. In future we analyze rainfall prediction by utilizing the parameters like $\mathrm{Co}_{2}$, hydrogen, nitrogen contents in air and corresponding land area whether the chance of rainfall is there or not.

\section{References}

1. http://www.colorado.edu/geography/gcraft/ notes/remote/remote_f.html

2. https://prezi.com/i1hrip789ruy/orbits-andsatellites.

3. http://www.oneonta.edu/faculty/baumanpr/ geosat2/RS\%20History\%20II/RS-HistoryPart-2.html

4. Harshaverdana "Aadoption of Hadoop for a remote sensing real time big data analysis" international Journal of Advance Research in Engineering, Science \&Technology e-ISSN: 2393 -9877, p- ISSN: 2394-2444, Volume 3, Issue 6, June-2016.

5. J. Shi Jiaji Wu, Anand Paul, Licheng Jiao, Maoguo Gong "Change detection in synthetic aperture radar image based on fuzzy active contour" Mathematical Problems in Engineering Volume 2014 (2014), Article ID 870936, http://dx.doi. org/10.1155/2014/870936.
6. Sheik Mujabar, N. Chandrasekar "Coastal erosion hazard and vulnerability assessment for southern coastal Tamil Nadu of India by using remote sensing and GIS. Nat Hazards. 69(3):1295-314. (2013)

7. Chu, X.G.Sun, S.K.Zhai, K.H.Xu "Changing pattern of accretion/erosion of the modern Yellow River (Huanghe) subaerial delta, China: Based on remote sensing images. Mar Geol. 227(1-2):13-30 (2006)

8. Cowart L., D. Reide Corbett and J.P. Walsh "Shoreline change along sheltered coastlines: Insights from the neuse river estuary, NC, USA. Remote Sens. 3(7):1516-34 (2011)

9. RUHOFF A.L. "Modelagem ambiental com a simulação de cenários preservacionistas. 93 f. Dissertação (Mestrado em Geomática) Universidade Federal de Santa Maria, Santa Maria, 2004. 